\title{
Some new quantum Trapezoid and quantum Midpoint type inequalities for preinvex functions
}

\author{
Muhammad Aamir Ali ${ }^{1}$ and Hüseyin BUDAK ${ }^{2}$ \\ ${ }^{1}$ Nanjing Normal University School of Mathematical Sciences \\ ${ }^{2}$ Düzce University
}

December 8, 2020

\begin{abstract}
In this article, we derive Hermite-Hadamard inequalities for preinvex functions using the quantum integrals and show their validation with mathematical examples. We prove midpoint and trapezoidal inequalities for $\mathrm{q}^{\wedge}\{2\}$-differentiable preinvex functions via $\mathrm{q}^{\wedge}\{2\}$-quantum integrals. Moreover, we show with an example that the already proved inequality of HermiteHadamard type for preinvex functions via q- $\{1\}$-quantum integrals is not valid for preinvex functions and we give its correct version. We prove the midpoint inequalities for $\mathrm{q}_{-}\{1\}$-differentiable preinvex functions via $\mathrm{q}_{-}\{1\}$-quantum integrals. It is also shown that the newly proved results transformed into some known results by considering the limit $\mathrm{q}-1-$ and $\eta(2,1)=-\eta(1,2)=$ 2- 1 in the newly derived results.
\end{abstract}

\section{Hosted file}

MMAS1222020.pdf available at https://authorea.com/users/330633/articles/497718-some-newquantum-trapezoid-and-quantum-midpoint-type-inequalities-for-preinvex-functions 